\title{
Editorial
}

\section{Josef Schrader}

(C) Der/die Autor(en) 2016. Dieser Artikel ist eine Open-Access-Publikation.

Seit dem Jahr 2015 arbeitet die „Zeitschrift für Weiterbildungsforschung“ (ZfW) auf der Grundlage eines veränderten Konzepts: Nicht mehr nur die Herausgebergruppe spricht Einladungen zu je aktuellen Themen der Forschung zur Erwachsenen- und Weiterbildung aus, vielmehr werden auch Gastherausgeberinnen und Gastherausgeber für Themenhefte gewonnen. Die Gastherausgeber werden gebeten, Konzepte für Themenhefte einzureichen, die von den Herausgebern begutachtet und begleitet werden. In der Regel stützen sich diese Konzepte bereits auf eine erste Sichtung aktueller Forschungsarbeiten, so dass gezielt Autorinnen und Autoren auf das Themenheft aufmerksam gemacht werden können. Zudem bemühen sich die Herausgeber, jedes Themenheft mit einem so genannten Fokus-Beitrag einzuleiten, der einen Überblick über den einschlägigen Forschungsstand gibt, um die gedruckten Beiträge vor diesem Hintergrund verorten zu können. Darüber hinaus erfolgt weiterhin wie bisher ein offener „Call for papers“. Dieses Konzept hat sich nach unseren bisherigen Erfahrungen bewährt; dies zeigt sich u. a. an der Zahl der eingereichten und positiv begutachteten Beiträge in den beiden letzten Jahren.

Über die direkte oder offene Einladung zu Themenheften hinaus bietet die ZfW weiterhin die Möglichkeit, so genannte „freie“ Beiträge einzureichen, die sich nicht den Schwerpunkten der Themenhefte zuordnen lassen. Damit wollen wir Raum schaffen, um die Vielfalt aktueller Forschungsschwerpunkte der Erwachsenen- und Weiterbildung möglichst breit abdecken zu können. Diese Beiträge werden dann zumeist in der Rubrik „Forum“ veröffentlicht, die das Thema des jeweiligen Heftes ergänzt.

\footnotetext{
J. Schrader $(\bowtie)$

Deutsches Institut für Erwachsenenbildung, Leibniz-Zentrum für Lebenslanges Lernen, Bonn, Deutschland

E-Mail: schrader@die-bonn.de
} 
Da im Laufe der vergangenen Monate eine größere Anzahl freier Beiträge positiv begutachtet wurde, haben wir uns entschlossen, das dritte Heft im Jahr 2016 ohne thematischen Fokus zu gestalten. Bereits ein erster Blick auf die hier dokumentierten Beiträge zeigt, dass sie Schwerpunkte der Forschung zur Erwachsenen- und Weiterbildung repräsentieren, die sich in den vergangenen Jahren abgezeichnet, aber noch nicht zu Themenheften geführt haben. Dies betrifft z. B. die Organisationsforschung oder die Forschung zur Professionalisierung des Lehrpersonals. Auffallend ist, dass es sich bei den hier veröffentlichten Beiträgen durchgehend um empirische Arbeiten handelt, die in der Mehrzahl aus Qualifikationsprojekten hervorgegangen sind. Eine weitere Gemeinsamkeit besteht darin, dass sie zumeist auf das berufliche Handeln unterschiedlicher Gruppen von Beschäftigten in der Erwachsenen- und Weiterbildung gerichtet sind.

Dem in der Erwachsenen- und Weiterbildung bereits seit vielen Jahren gut etablierten und weiterhin wachsenden Bereich der Organisationsforschung lässt sich der erste Beitrag zuordnen. Dörthe Herbrechter wendet sich darin unter dem Titel „Im Schatten der Hierarchie“ der Frage zu, wie Organisationen der Weiterbildung geleitet werden. Die Autorin betont, dass die bisherigen Forschungsergebnisse zeigen, dass das Managementhandeln in Weiterbildungseinrichtungen nicht allein betriebswirtschaftlichen Effektivitäts- und Effizienzvorstellungen folgt, sondern auch und maßgeblich durch das jeweilige Bildungsverständnis und die organisationalen Rahmenbedingungen bestimmt ist. Vor diesem Hintergrund geht der Beitrag der Frage nach, welches Führungsverständnis und welche damit verbundenen Handlungsorientierungen Führungspersonen in Einrichtungen der Weiterbildung entwickeln, wie sie durch ihr Führungsverständnis und entsprechende Handlungsorientierungen hierarchische Formen der Koordination befördern und inwieweit sie ,im Schatten der Hierarchie“ weitere Formen der Handlungskoordination etablieren. Empirisch arbeitet die Autorin mit Fallstudien aus Einrichtungen der Weiterbildung, die in unterschiedlichen Kontexten agieren. Der vorgestellte Fallvergleich deutet darauf hin, dass Leitungspersonen in Weiterbildungsorganisationen durch ihr Führungsverständnis und ihre entsprechenden Handlungsorientierungen sowohl hierarchische als auch nicht-hierarchische Formen des koordinierten Zusammenwirkens ihrer Mitarbeitenden unterstützen. Inwieweit dabei institutionelle, organisationale oder personale Einflussfaktoren zum Ausdruck kommen, weist die Autorin als weiteren Forschungsbedarf aus.

Auch der Beitrag von Michael Krüger lässt sich der Organisationsforschung zuordnen. Das Forschungsinteresse ist auf das Wissen gerichtet, das in Studiengängen zum Bildungsmanagement präsentiert wird. Denn mit der Etablierung neuer Steuerungsmodelle und gestiegenen Erwartungen an den ökonomischen Erfolg von Organisationen der Weiterbildung ist das Bildungsmanagement ins Blickfeld einer breit angelegten Debatte in Praxis, Öffentlichkeit und Wissenschaft geraten, zumeist komplementär, teils aber auch übergreifend zu den bereits seit Längerem etablierten Aufgaben der Programmplanung und Angebotsentwicklung. Der Beitrag präsentiert Befunde einer empirischen Studie, die auf die curricularen Inhalte, hier als „Lernwissen“ bezeichnet, von insgesamt ca. 50 Masterstudiengängen zum Bildungsmanagement im deutschsprachigen Raum gerichtet war. Die Studiengänge adressieren verschiedene Bildungsbereiche von der frühen Bildung über Schule und 
Hochschule bis zur Weiterbildung. Der Autor identifiziert auf der Grundlage der Analyse von Modulhandbüchern und ergänzender Experteninterviews Studiengänge, die keine spezifische Schwerpunktsetzung ausweisen, und Studiengänge, die mal auf Forschung, mal auf Leadership und mal auf Management ausgerichtet sind.

Drei weitere Beiträge beschäftigen sich mit dem Lehrpersonal in der Erwachsenenbildung und damit mit einem Forschungsgegenstand, der in den vergangenen Jahren ebenfalls erhöhte Aufmerksamkeit gefunden hat. Im Mittelpunkt der Arbeiten stehen die (professionellen) Kompetenzen des Lehrpersonals. Tim Stanik beschäftigt sich in seinem Beitrag mit einem empirischen Desiderat der Lehr-Lernforschung in der Erwachsenenbildung, dem mikrodidaktischen Planen von Lehrveranstaltungen. In seinem Beitrag sichtet er zunächst den konzeptuellen und empirischen Stand der erwachsenenbildnerischen, aber auch der schulpädagogischen Forschung. Mikrodidaktisches Planungshandeln wird danach als ein komplexer Entscheidungsprozess verstanden. Die Sichtung des Forschungsstandes führt zu Entscheidungsfeldern und potentiellen Einflussfaktoren auf das mikrodidaktische Planungshandeln. Auf dieser Grundlage skizziert der Autor das Design einer empirischen Studie, um sowohl die expliziten als auch die impliziten Faktoren des Planungshandelns zu analysieren. Gestützt auf problemzentrierte Interviews und die Analyse von Planungsentwürfen soll eine materiale Theorie des Planungshandelns entwickelt werden. Der Autor geht davon aus, dass mikrodidaktisches Planungshandeln als kognitive, materiale und soziale Praxis interpretiert werden muss, in der implizites Erfahrungswissen, deklaratives Professionswissen und auch (biografisch geprägte) Auffassungen über fachliche Inhalte, subjektive Rollenverständnisse, Wissenskonzepte als Lehrende, stereotype Konstruktionen der Teilnehmenden sowie Einflüsse der Weiterbildungsorganisationen eine Rolle spielen.

$\mathrm{Ob}$ und gegebenenfalls wie digitale Medien das zumeist als komplex und interdependent beschriebene mikrodidaktische Planungshandeln von (angehenden) Lehrkräften der Erwachsenen- und Weiterbildung unterstützen können, wird in einem Beitrag von Josef Schrader und Sabine Schöb untersucht. Vorgestellt wird die Entwicklung und Begründung einer neu entwickelten Kursplanungs-App, die (angehende) Lehrkräfte der Erwachsenen- und Weiterbildung bei der Planung von Anfangssituationen unterstützen soll. Insbesondere zielt die App darauf, das Arbeitsgedächtnis der Planenden zu entlasten, indem das Aufzeigen von Interdependenzbeziehungen zwischen einer Vielzahl didaktischer Dimensionen von der App übernommen wird, indem multimodal gestaltete Informationen zu Unterrichtsmethoden mithilfe von Videos zur Anschaulichkeit beitragen und indem Unterrichtsentwürfe iterativ und spielerisch erarbeitet werden können. Damit sollen kognitive Kapazitäten für didaktische Reflexionen im engeren Sinne „frei“ werden, so dass die Qualität der Entwürfe verbessert werden kann. Erste empirische Befunde aus einer evaluativen Studie zeigen, dass dieses digitale Tool didaktisch begründete Entscheidungen von Lehrenden unterstützen und damit zu einer größeren Professionalität des Lehrhandelns beitragen kann.

Lisa David schließlich beschäftigt sich in einer professionstheoretischen Perspektive mit der Reflexion des beruflichen Handelns als einer zentralen Anforderung von Kursleiterinnen und Kursleitern. Ob dies auch geleistet wird bzw. geleistet werden kann, ist bislang wenig untersucht. Die Autorin unterscheidet zum einen die direkte 
didaktische Reflexion als Nachdenken über das eigene Lehrhandeln bzw. die LehrLernsituation, zum anderen eine Reflexion, die auch das berufliche Selbstverständnis einschließt. Dabei geht es u. a. um die Frage, wie subjektive Einschätzungen über den Erfolg von Lehr-Lernprozessen den jeweils gesteckten Reflexionsrahmen beeinflussen. Deutlich wird in der Befragungsstudie, dass Kursleiterinnen und Kursleiter sehr unterschiedliche didaktisch-methodische Kategorien fokussieren, die eng mit den subjektiven Theorien der Lehrkräfte über gelingende Lehr-Lernprozesse zusammenhängen. Dabei werden sehr unterschiedliche Erfolgskriterien bzw. deren (Nicht-)Erreichung zum Anlass von Selbstreflexion genommen. Dies geschieht insgesamt eher selten und eher im Misserfolgs- als im Erfolgsfall. Die Autorin sieht darin auch eine Strategie der Lehrkräfte zur Sicherung der Fortführung der Arbeit unter den Bedingungen empfundener Unsicherheit, zumal Standards der Berufsausübung fehlen.

Der abschließende Beitrag zur Hochschulbildung in Polen von Danuta Wajsprych schließlich befasst sich mit der Frage, welche Bedeutung Anerkennung für studierende Erwachsene hat. Den empirischen Hintergrund bildet eine Ausweitung staatlicher und privater universitärer Studienangebote in Polen seit dem Ende der 1980erJahre, die mit einer enormen Steigerung der Studierendenzahlen einherging. Die empirischen Befunde der Autorin stützen sich auf eine Befragung von so genannten nicht-traditionellen Studierenden in Fernstudiengängen an der Hochschule Olsztyn. Theoretisch stützt sich die Autorin auf die Arbeiten von Axel Honneth, der Anerkennung als eine konstitutive Voraussetzung von Gesellschaftlichkeit interpretiert. Honneth betrachtet Liebe, Recht und Solidarität als Voraussetzungen für Selbstvertrauen, Selbstachtung und Selbstwertgefühl. Während die Studierenden in der Befragung häufig eine tiefe Zufriedenheit mit ihren Studienerfahrungen zum Ausdruck bringen, die sich in einem Wachstum des Selbstvertrauens und einem gesteigerten Selbstwertgefühl zeigen, fragt die Autorin nach dem Spannungsfeld von „naiver“ und ,tatsächlicher“ Emanzipation.

Die ,Zeitschrift für Weiterbildungsforschung“ wird auch in den kommenden Jahren regelmäßig thematisch offene Hefte mit einer Sammlung ,freier“ Beiträge anbieten. Dies werden wir tun, um einerseits die Vielfalt laufender Forschungsarbeiten in der Erwachsenenbildung repräsentieren zu können, andererseits aber auch, um die Wartezeiten zwischen der Einreichung und der Publikation von Beiträgen zu verkürzen, insbesondere dann, wenn die Zahl der frei eingereichten und positiv begutachteten Beiträge so groß ist, dass sie nicht mehr in Themenheften untergebracht werden können. Wir hoffen damit, sowohl den Interessen der Autorinnen und Autoren als auch jenen der Leserinnen und Leser gerecht zu werden.

Open Access Dieser Artikel wird unter der Creative Commons Namensnennung 4.0 International Lizenz (http://creativecommons.org/licenses/by/4.0/deed.de) veröffentlicht, welche die Nutzung, Vervielfältigung, Bearbeitung, Verbreitung und Wiedergabe in jeglichem Medium und Format erlaubt, sofern Sie den/die ursprünglichen Autor(en) und die Quelle ordnungsgemäß nennen, einen Link zur Creative Commons Lizenz beifügen und angeben, ob Änderungen vorgenommen wurden. 